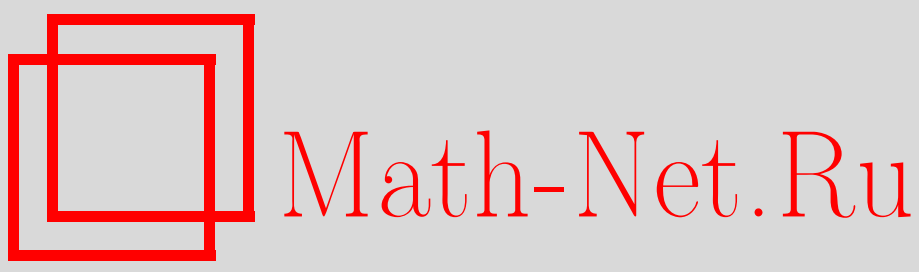

Д. Н. Иванов, О сбалансированных системах идемпотентов, Матем. сб., 2001, том 192, номер 4, 73-86

DOI: https://doi.org/10.4213/sm558

Использование Общероссийского математического портала Math-Net.Ru подразумевает, что вы прочитали и согласны с пользовательским соглашением

http: //www.mathnet.ru/rus/agreement

Параметры загрузки:

IP : 54.196 .121 .252

26 апреля 2023 г., $10: 30: 18$ 


\title{
Д.Н. Иванов
}

\section{О сбалансированных системах идемпотентов}

\begin{abstract}
Сбалансированньм базисом ассоциативной полупростой конечномерной над полем комплексных чисел $\mathbb{C}$ алгебры называется система идемпотентов $\left\{e_{i}\right\}$, образуюшая линейный базис и такая, что $\operatorname{Tr} e_{i}$ и $\operatorname{Tr} e_{i} e_{j}$ не зависят от $i, j, i \neq j$, где $\operatorname{Tr}$ - след регулярного представления алгебры. В статье строятся сбалансированные базисы в алгебре матриц $\mathrm{M}_{p^{n}}(\mathbb{C}), p$ нечетное простое. Для алгебр матриц такие базисы до сих пор были известны лишш в случае $\mathrm{M}_{2}(\mathbb{C})$ и $\mathrm{M}_{3}(\mathbb{C})$. Доказывается несуществование сбалансированных базисов определенных рангов, обладающих регулярной элементарной абелевой 2-группой автоморфизмов, в алгебрах $\mathrm{M}_{2^{n}}(\mathbb{C}), n>1$. Кроме того, классифицируются сбалансированные 1-системы из $n+1$ идемпотента ранга $r$ в алгебре $\mathrm{M}_{r n}(\mathbb{C})$.
\end{abstract}

Библиография: 8 названий.

\section{§1. Введение}

В работе [1] было введено понятие сбалансированной системы идемпотентов в ассоциативной полупростой конечномерной над полем комплексных чисел ${ }^{1} \mathbb{C}$ алгебре А. Это понятие получило свое развитие в работе [2]. Напомним основное определение.

ОПРЕДЕЛЕНИЕ. Пусть система идемпотентов $\mathscr{E}=\left\{e_{1}, \ldots, e_{v}\right\}$ в алгебре $\mathbf{A}$ удовлетворяет следующим условиям:

1) (условие сбалансированности) $e_{1}+\cdots+e_{v}=k \cdot \mathbf{1}_{A}$, где $\mathbf{1}_{A}$ - единичный элемент алгебры $\mathbf{A}, k \in \mathbb{C}$;

2) (условие равноугольности) $\operatorname{Tr} e_{i_{1}} \cdots e_{i_{s}}=\lambda_{s}$ для любых различных индексов $i_{1}, \ldots, i_{s}, s=1, \ldots, t$, где $\operatorname{Tr}$ - след регулярного представления алгебры $\mathbf{A}$ и $\lambda_{s}$ зависит только от $s$.

Тогда Еீ называется сбалансированной $t$-системой, или просто $t$-системой.

Если $\mathscr{E}$ - сбалансированная 2-система, образующая линейный базис в $\mathbf{A}$, то $\mathscr{E}$ называется сбалансированныц базисом. Для $\sigma \in$ Aut $\mathbf{A}$ система $\mathscr{E} \sigma=\left\{e_{i}^{\sigma}\right\}$ является сбалансированной $t$-системой, сопряженной к $\mathscr{E}$. Группа Aut $\mathscr{E}=\{\sigma \in$ Aut $\left.\mathbf{A}: \mathscr{E}^{\sigma}=\mathscr{E}\right\}$ называется группой автоморфизмов системы $\mathscr{E}$. Подгруппы в Aut $\mathscr{E}$ также будут называться группами автоморфизмов. Из контекста всегда

Работа выполнена при частичной поддержке Российского фонда фундаментальных исследований (грант № 99-01-00233).

${ }^{1} \mathrm{C}$ равньм успехом можно вместо $\mathbb{C}$ рассматривать произвольное алгебраически замкнутое поле характеристики 0 .

(C) Д.Н. ИвАнОВ 2001 
будет ясно, о чем идет речь. Система $\widehat{\mathscr{E}}=\left\{\widehat{e}_{i}=\mathbf{1}_{A}-e_{i}\right\}$ называется дополнительной к $\mathscr{E}$. Очевидно, что $\widehat{\mathscr{E}}$, как и $\mathscr{E}$, является $t$-системой и имеет ту же самую группу автоморфизмов.

Как отмечалось в [1], система $\mathscr{E}$, удовлетворяющая условию равноугольности для 2-систем, линейно независима, и если $\mathscr{E}$ является базисом, то условие сбалансированности для $\mathscr{E}$ выполняется автоматически. Заметим, что согласно [1] не существует 3-систем, являющихся базисами.

Параметр $\lambda_{1}=\operatorname{Tr} e_{i}$ будем обозначать через $r$ и назьвать рангом идемпотентов и всей $t$-системы. Для $t$-системы $\mathscr{E}$ ранга $r$ из $v$ идемпотентов будем использовать обозначение $t$ - $(v, r)$-систем $a$. В случае матричной алгебры $\mathbf{A}=\mathrm{M}_{n}(\mathbb{C})=\mathrm{M}_{n}$ под рангом будем понимать обычный ранг матрицы и для $\mathscr{E}$ употреблять обозначение $t$ - $(v, r, n)$-система.

Понятие сбалансированной $t$-системы идемпотентов было введено как обощение понятия комбинаторных $t$-схем, которые соответствуют в этом контексте случаю коммутативных алгебр А. Например, $2-(v, k, \lambda)$-схеме (по поводу обозначений cм. [3]) отвечает 2-( $v, r)$-система в коммутативной алгебре $b \mathrm{M}_{1}(\mathbb{C})=\mathbb{C} \oplus \cdots \oplus \mathbb{C}$ ( $b$ слагаемых), где $b$ - число блоков схемы, $r$ - число появлений точки в разных блоках, $\lambda=\lambda_{2}$ - число появлений двух точек в разных блоках, $k$ - мощность блока, равная коэффициенту при $\mathbf{1}_{A}$ в условии сбалансированности. Отметим, что симметричньм 2-схемам отвечают сбалансированные базисы.

Алгебру, допускающую сбалансированный базис, будем называть сбалансированной. До сих пор были известны следующие примеры некоммутативных сбалансированных алгебр. В [1], [2] были построены сбалансированные базисы в алгебрах $\mathrm{M}_{2}(\mathbb{C})$ и $\mathrm{M}_{3}(\mathbb{C})$. Кроме того, в [1] доказано, что существование сбалансированного базиса ранга $n+1$ в алгебре $(n+1) \mathrm{M}_{1} \oplus \mathrm{M}_{n}$ равносильно сушествованию однородного ортогонального разложения типа $n \mathrm{M}_{1}$ алгебры $\mathrm{M}_{n}$, которое, в свою очередь, равносильно существованию ортогонального разложения простой алгебры Ли типа $A_{n-1}$ в сумму картановских подалгебр (см. об этих разложениях [4]). Таким образом, в силу результатов работы [4] алгебра $(n+1) \mathrm{M}_{1} \oplus \mathrm{M}_{n}$ при $n=p^{m}, p$ простое, сбалансирована.

Основной целью данной работы является построение сбалансированных базисов в алгебрах матриц $\mathrm{M}_{p^{n}}$, где $p$ нечетное простое. Группы автоморфизмов этих базисов содержат нормальную регулярную элементарную абелеву $p$-подгруппу. Также доказывается несушествование сбалансированных базисов ранга $r$ в алгебpe $\mathrm{M}_{2^{n}}(\mathbb{C}), n>1$, обладающих регулярной элементарной абелевой 2-группой автоморфизмов, если $\frac{r\left(2^{n}-r\right)}{2^{2 n}-1}$ не является квадратом рационального числа. Таким образом, данная работа открывает исследования сбалансированных базисов с регулярной группой автоморфизмов в некоммутативных алгебрах. Кроме того, классифицируются 1- $(n+1, r)$-системы в алгебре $\mathrm{M}_{r n}$, что обобшает полученную в [2] классификацию таких систем в случае $r=1$.

За стандарт обозначений, касающихся конечных групп, принята книга [5]. Так, $G=A . B$ обозначает расширение группы $A$ посредством $B \cong G / A ; G=A: B$ - расщепляемое расширение; $p^{n}$ - элементарная абелева группа ранга $n ; p^{1+2 n}-$ экстраспециальная группа.

Автор выражает глубокую благодарность А.И. Кострикину за поддержку и 
внимание к работе.

\section{§2. Матрицы $J_{u}$}

Пусть $p$ простое, $\varepsilon$ - примитивный корень степени $p$ из 1. Определим матрицы размера $p \times p$ :

$$
D=\left(\begin{array}{cccc}
1 & & & \\
& \varepsilon & & \\
& & \ddots & \\
& & & \varepsilon^{p-1}
\end{array}\right), P=\left(\begin{array}{ccccc}
0 & 0 & \ldots & 0 & 1 \\
1 & 0 & \ldots & 0 & 0 \\
0 & 1 & \ldots & 0 & 0 \\
\ldots & \ldots \ldots & \ldots & \ldots \\
0 & 0 & \ldots & 1 & 0
\end{array}\right), \quad J_{a, b}=D^{a} P^{b}
$$

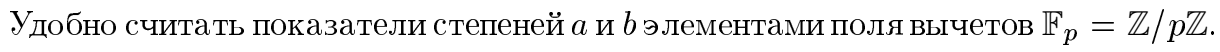
Далее, положим

$$
J_{u}=J_{a_{1}, b_{1}} \otimes \cdots \otimes J_{a_{n}, b_{n}}, \quad u=\left(a_{1}, \ldots, a_{n} ; b_{1}, \ldots, b_{n}\right) \in \mathbb{F}_{p}^{2 n} .
$$

Так как $D^{a} P^{b}=\varepsilon^{a b} P^{b} D^{a}$, то имеем следующие формулы:

$$
\begin{gathered}
J_{u} J_{u^{\prime}}=\varepsilon^{B\left(u, u^{\prime}\right)} J_{u+u^{\prime}}, \quad \text { где } B\left(u, u^{\prime}\right)=-\sum_{i=1}^{n} a_{i}^{\prime} b_{i}, \\
J_{u} J_{v} J_{u}^{-1}=\varepsilon^{\langle u \mid v\rangle} J_{v}, \quad \text { где }\left\langle u \mid u^{\prime}\right\rangle=\sum_{i=1}^{n}\left(a_{i} b_{i}^{\prime}-a_{i}^{\prime} b_{i}\right)=B\left(u, u^{\prime}\right)-B\left(u^{\prime}, u\right) .
\end{gathered}
$$

Отметим, что $\langle u \mid v\rangle$ - невырожденная кососимметрическая форма на $\mathbb{F}_{p}^{2 n}$. Так как

$$
J_{u}^{k}=\varepsilon^{\frac{1}{2} k(k-1) B(u, u)} J_{k u},
$$

TO

$$
J_{u}^{p}=E \text { при } p>2 \text { и } J_{u}^{2}=(-1)^{B(u, u)} E \text { при } p=2,
$$

где $E$ - единичная матрица. Кроме того,

$$
J_{u}^{-1}=\varepsilon^{B(u, u)} J_{-u}, \quad J_{u}^{t}=\varepsilon^{-B(u, u)} J_{\widehat{u}}, \quad \bar{J}_{u}=J_{-\widehat{u}},
$$

где черта над матрицей означает комплексное сопряжение и $\widehat{u}=\left(a_{1}, \ldots, a_{n} ;-b_{1}\right.$, $\left.\ldots,-b_{n}\right)$. Отметим, что $B(\widehat{u}, \widehat{u})=-B(u, u)$.

Положим

$$
\begin{aligned}
N(J) & =\left\{\sigma \in \text { Aut } \mathrm{M}_{p^{n}}:\left\langle J_{u}^{\sigma}\right\rangle_{\mathbb{C}}=\left\langle J_{u^{\prime}}\right\rangle_{\mathbb{C}}\right\} \\
H & =\left\{\text { сопряжения матрицами } J_{u}, u \in \mathbb{F}_{p}^{2 n}\right\} .
\end{aligned}
$$

Группа $H$ является нормальной элементарной абелевой $p$-подгруппой ранга $2 n$ в групше $N(J)$. Как установлено в [6], фактор-группа $N(J) / H$ изоморфна симплектической группе $S p_{2 n}(p)$. Расширение $N(J)=H . S p_{2 n}(p)$ нерасщепляемо только 
при $p=2$ и $n>2$. При $p>2$ группа $S p_{2 n}(p)$ вкладывается в $N(J)$ путем отображения $\phi \mapsto \Phi_{\phi}$, где

$$
\Phi_{\phi}\left(J_{u}\right)=\varepsilon^{\frac{1}{2}(B(\phi(u), \phi(u))-B(u, u))} J_{\phi(u)}
$$

(см. [7]).

Легко видеть, что группа, порожденная матрицами $J_{u}$, реализует неприводимое представление экстраспециальной группы $p_{+}^{1+2 n}$ экспоненты $p$ в случае $p>2$ и экстраспециальной группы $2_{+}^{1+2 n} \cong D_{4} * \cdots * D_{4}(n$ множителей $)$ в случае $p=2$, где $D_{4}$ - группа диэдра порядка 8. Матрицы $J_{u}$ имеют множество применений. В частности, они использовались для построения ортогональных разложений простых алгебр Ли типа $A_{p^{n}-1}$ в сумму картановских подалгебр [4], [7].

\section{§ 3. Сбалансированные базисы в $\mathrm{M}_{p^{n}}(\mathbb{C}), p>2$}

Положим

$$
A_{x}=\frac{1}{p^{n}} \sum_{u} \varepsilon^{\langle u \mid x\rangle+\frac{1}{2} B(u, u)} J_{u}, \quad x \in \mathbb{F}_{p}^{2 n},
$$

где суммирование ведется по всем $u=\left(a_{1}, \ldots, a_{n} ; b_{1}, \ldots, b_{n}\right) \in \mathbb{F}_{p}^{2 n}$.

Teоpema 1. 1) $A_{x} A_{y}=\varepsilon^{c(x, y)} J_{2(y-x)}$, zдe $c(x, y)=2(B(x, x)+B(y, y)-$ $2 B(x, y))$, в частности, $A_{x}^{2}=J_{0}=E-$ единичная матрича;

2) системы идемпотентов

$$
\mathscr{B}_{p^{n}}^{+}=\left\{e_{x}^{+}=\frac{1}{2}\left(E+A_{x}\right): x \in \mathbb{F}_{p}^{2 n}\right\}, \quad \mathscr{B}_{p^{n}}^{-}=\left\{e_{x}^{-}=\frac{1}{2}\left(E-A_{x}\right): x \in \mathbb{F}_{p}^{2 n}\right\}
$$

являются дополнительными сбалансированными базисами алгебры $\mathrm{M}_{p^{n}}$ рангов $\frac{p^{n}+1}{2} u \frac{p^{n}-1}{2}$ соответственно;

3) Aut $\mathscr{B}_{p^{n}}^{+}=$Aut $\mathscr{B}_{p^{n}}^{-}=N(J) \cong p^{2 n}: S p_{2 n}(p)$.

ДокАЗАТЕльство. 1) Нам понадобится следуюший простой факт о тригонометрических суммах:

$$
\sum_{a \in \mathbb{F}_{p}} \varepsilon^{a b}= \begin{cases}0, & \text { если } b \neq 0 \\ p, & \text { если } b=0\end{cases}
$$

Пусть $u=\left(a_{1}, \ldots, a_{n} ; b_{1}, \ldots, b_{n}\right), x=\left(f_{1}, \ldots, f_{n} ; g_{1}, \ldots, g_{n}\right)$. Тогда в силу $(3.1)$ имеем

$$
\begin{aligned}
\sum_{u} \varepsilon^{\langle u \mid x\rangle} & =\sum_{u} \varepsilon^{a_{1} g_{1}+\cdots+a_{n} g_{n}-b_{1} f_{1}-\cdots-b_{n} f_{n}} \\
& =\left(\sum_{a_{1}} \varepsilon^{a_{1} g_{1}}\right) \ldots\left(\sum_{b_{n}} \varepsilon^{-b_{n} f_{n}}\right)= \begin{cases}0, & \text { если } x \neq 0 \\
p^{2 n}, & \text { если } x=0 .\end{cases}
\end{aligned}
$$


Используя формулу (2.1), получаем

$$
\begin{aligned}
A_{x} A_{y} & =\frac{1}{p^{2 n}} \sum_{u, v} \varepsilon^{\langle u \mid v\rangle+\langle v \mid y\rangle+\frac{1}{2} B(u, u)+\frac{1}{2} B(v, v)+B(u, v)} J_{u+v} \\
& =\frac{1}{p^{2 n}} \sum_{v} \sum_{u} \varepsilon^{\langle u \mid x\rangle+\langle v-u \mid y\rangle+\frac{1}{2} B(u, u)+\frac{1}{2} B(v-u, v-u)+B(u, v-u)} J_{v} \\
& =\frac{1}{p^{2 n}} \sum_{v} \sum_{u} \varepsilon^{\langle u \mid x-y\rangle+\langle v \mid y\rangle+\frac{1}{2} B(v, v)+\frac{1}{2}\langle u \mid v\rangle} J_{v} \\
& =\frac{1}{p^{2 n}} \sum_{v}\left(\sum_{u} \varepsilon^{\left\langle u \mid x-y+\frac{1}{2} v\right\rangle}\right) \varepsilon^{\langle v \mid y\rangle+\frac{1}{2} B(v, v)} J_{v} .
\end{aligned}
$$

В силу (3.2) в заключительном выражении (3.3) единственный отличный от 0 коэффициент будет при $J_{v}$, где $v=2(y-x)$. Этот коэффициент равен

$$
\frac{1}{p^{2 n}} p^{2 n} \varepsilon^{\langle 2(y-x \mid y)\rangle+\frac{1}{2} B(2(y-x), 2(y-x))}=\varepsilon^{2(B(x, x)+B(y, y)-2 B(x, y))} .
$$

2) Так как $A_{x}^{2}=E$, то $\frac{1}{2}\left(E \pm A_{x}\right)$ суть идемпотенты. Далее,

$$
\operatorname{tr} J_{u}=0 \text { при } u \neq 0 \Rightarrow \operatorname{tr} A_{x}=1 \Rightarrow \operatorname{tr} \frac{1}{2}\left(E \pm A_{x}\right)=\frac{p^{n} \pm 1}{2} .
$$

Кроме того, в силу 1) $\operatorname{tr} A_{x} A_{y}=0$ при $x \neq y$. Значит, $\operatorname{tr} e_{x}^{ \pm} e_{y}^{ \pm}=\operatorname{tr} \frac{1}{4}\left(E \pm A_{x} \pm\right.$ $\left.A_{y}+A_{x} A_{y}\right)=\frac{1}{4}\left(p^{n} \pm 2\right)$. Отсюда следует, что $\mathscr{B}_{p^{n}}^{ \pm}$удовлетворяют условию равноугольности для 2-систем.

С помощю определителя Грама легко доказать линейную независимость систем $\mathscr{B}_{p^{n}}^{ \pm}$. Значит, $\mathscr{B}_{p^{n}}^{ \pm}$суть базисы. Раскладывая единичную матрицу $E$ по этим базисам, получаем, что выполняется условие сбалансированности.

Также условие сбалансированности можно установить непосредственно, используя формулу (3.2):

$$
\sum_{x} A_{x}=\frac{1}{p^{n}} \sum_{x} \sum_{u} \varepsilon^{\langle u \mid x\rangle+\frac{1}{2} B(u, u)} J_{u}=\frac{1}{p^{n}} \sum_{u}\left(\sum_{x} \varepsilon^{\langle u \mid x\rangle}\right) \varepsilon^{\frac{1}{2} B(u, u)} J_{u}=p^{n} E .
$$

Так как сумма $A_{x}$ - скалярная матрица, то это же можно сказать и о сумме $e_{x}^{ \pm}$.

3) Очевидно, Aut $\mathscr{B}_{p^{n}}^{+}=$Aut $\mathscr{B}_{p^{n}}^{-}$. Обозначим Aut $\mathscr{B}_{p^{n}}^{+}=G$. Группа $G$ переставляет идемпотенты $e_{x}^{+}$. Значит, $G$ переставляет инволюции $A_{x}$. Так как $A_{x} A_{y}=\varepsilon^{c(x, y)} J_{2(y-x)}$, то $G$ переставляет прямые $\left\langle J_{u}\right\rangle_{\mathbb{C}}$. Значит, $G$ содержится в $N(J)$. С другой стороны, в силу (2.2) и (2.4) имеем

$$
\begin{aligned}
J_{v} A_{x} J_{v}^{-1} & =\frac{1}{p^{n}} \sum_{u} \varepsilon^{\langle u \mid x\rangle+\frac{1}{2} B(u, u)+\langle v \mid u\rangle} J_{u}=A_{x-v} \\
\Phi_{\phi}\left(A_{x}\right) & =\frac{1}{p^{n}} \sum_{u} \varepsilon^{\langle u \mid x\rangle+\frac{1}{2} B(u, u)+\frac{1}{2}(B(\phi(u), \phi(u))-B(u, u))} J_{\phi(u)} \\
& =\frac{1}{p^{n}} \sum_{u} \varepsilon^{\langle\phi(u) \mid \phi(x)\rangle+\frac{1}{2} B(\phi(u), \phi(u))} J_{\phi(u)}=A_{\phi(x)} .
\end{aligned}
$$

Таким образом, $N(J)$ содержится в $G$, и мы получаем, что $G=N(J)$. Теорема доказана. 
ЗАмЕчАния. 1) Отметим, что базисы $\mathscr{B} \frac{ \pm}{p^{n}}$ состоят из эрмитовых матриц и инвариантны относительно операции транспонирования. Это вытекает из следующих равенств, полученных с учетом формул (2.3):

$$
\begin{aligned}
A_{x}^{t} & =\frac{1}{p^{n}} \sum_{u} \varepsilon^{\langle u \mid x\rangle+\frac{1}{2} B(u, u)} J_{u}^{t}=\frac{1}{p^{n}} \sum_{u} \varepsilon^{\langle u \mid x\rangle+\frac{1}{2} B(u, u)-B(u, u)} J_{\widehat{u}} \\
& =\frac{1}{p^{n}} \sum_{u} \varepsilon^{\langle\widehat{u} \mid-\widehat{x}\rangle+\frac{1}{2} B(\widehat{u}, \widehat{u})} J_{\widehat{u}}=A_{-\widehat{x}} ; \\
\bar{A}_{x} & =\frac{1}{p^{n}} \sum_{u} \varepsilon^{-\langle u \mid x\rangle-\frac{1}{2} B(u, u)} \bar{J}_{u}=\frac{1}{p^{n}} \sum_{u} \varepsilon^{-\langle u \mid x\rangle-\frac{1}{2} B(u, u)} J_{-\widehat{u}} \\
& =\frac{1}{p^{n}} \sum_{u} \varepsilon^{\langle-\widehat{u} \mid-\widehat{x}\rangle+\frac{1}{2} B(-\widehat{u},-\widehat{u})} J_{-\widehat{u}}=A_{-\widehat{x}} .
\end{aligned}
$$

2) Так как коэффициенты матриц систем $\mathscr{B}_{p^{n}}^{ \pm}$лежат в круговом поле $\mathbb{Q}[\varepsilon]$, то можно рассматривать действие на них автоморфизма $\sigma_{m} \in \operatorname{Gal}(\mathbb{Q}[\varepsilon] / \mathbb{Q})$, определенного равенством $\sigma_{m}(\varepsilon)=\varepsilon^{m}$. Оказывается, базисы $\mathscr{B}_{p^{n}}^{ \pm} \sigma_{m}$-инвариантны. Для $\sigma_{-1}$ это следует из эрмитовости матриц $e_{x}^{ \pm}$и инвариантности $\mathscr{B}_{p^{n}}^{ \pm}$относительно транспонирования, отмеченных выше. В случае произвольного $m$ это следует из равенств:

$$
\begin{gathered}
J_{u}^{\sigma_{m}}=J_{\alpha_{m}(u)}, \quad \text { где } \alpha_{m}(u)=\left(m a_{1}, \ldots, m a_{n} ; b_{1}, \ldots, b_{n}\right) \\
B\left(\alpha_{m}(u), \alpha_{m}(u)\right)=m B(u, u) ; \quad A_{x}^{\sigma_{m}}=\frac{1}{p^{n}} \sum_{u} \varepsilon^{m\langle u \mid x\rangle+\frac{m}{2} B(u, u)} J_{\alpha_{m}(u)} \\
=\frac{1}{p^{n}} \sum_{u} \varepsilon^{\left\langle\alpha_{m}(u) \mid \alpha_{m}(x)\right\rangle+\frac{1}{2} B\left(\alpha_{m}(u), \alpha_{m}(u)\right)} J_{\alpha_{m}(u)}=A_{\alpha_{m}(x)}
\end{gathered}
$$

3) Отметим, что группа $H$ действует регулярно на $\mathscr{B}_{p^{n}}^{ \pm}$. Разлагая произвольную матрицу по базису $\mathscr{B}_{p^{n}}^{ \pm}$, из транзитивности $H$ получаем, что $H$ действует неподвижно только на скалярных матрицах. Значит, $H$ проективно неприводима, т.е. линеаризация тождественного вложения $H \rightarrow \mathrm{Aut}_{p^{n}}$ является неприводимым представлением универсальной накрываюшей $\widehat{H}=p . H$. Кроме того, так как $S p_{2 n}(p)$ действует транзитивно на ненулевых векторах пространства $\mathbb{F}_{p}^{2 n}$, то $N(J)=H: S p_{2 n}(p)$ действует 2-транзитивно на $\mathscr{B}_{p^{n}}^{ \pm}$. Из указанных свойств $H$ и $N(J)$ вытекают нужные свойства систем $\mathscr{B}_{p^{n}}^{ \pm}$. Так, из проективной неприводимости $H$ следует условие сбалансированности, а из 2-транзитивности $N(J)$ следует условие равноугольности для 2-систем.

\section{§4. Сбалансированные базисы в $\mathrm{M}_{2^{n}}(\mathbb{C})$}

Сбалансированньй базис в $\mathrm{M}_{2}$ впервые был построен в [1], и там же была вычислена его группа автоморфизмов, которая оказалась изоморфной $A_{4}$. В [1] отмечалась единственность такого базиса с точностью до сопряженности. Докажем здесь это утверждение.

ТЕОРема 2. В $\mathrm{M}_{2}(\mathbb{C})$ существует единственный с точностью до сопряжсенности сбалансированный базис. 
ДокаЗАтельство. Ранг сбалансированного базиса $\left\{e_{0}, e_{1}, e_{2}, e_{3}\right\}$ в $\mathrm{M}_{2}$ может быть равен только 1. Из условий сбалансированности и равноугольности находим остальные параметры: $k=2, \lambda=\lambda_{2}=\frac{1}{3}$. Сопряжением мы можем добиться, чтобы $e_{0}=\operatorname{diag}(1,0)$. Тогда

$$
\operatorname{tr} e_{i} e_{0}=\frac{1}{3} \Rightarrow e_{i}=\frac{1}{3}\left(\begin{array}{c}
1 \\
x_{i}
\end{array}\right) \cdot\left(\begin{array}{ll}
1 & y_{i}
\end{array}\right), \quad \text { где } x_{i} y_{i}=2, \quad \text { так как } \operatorname{tr} e_{i}=1 .
$$

Далее, $\operatorname{tr} e_{i} e_{j}=\frac{1}{3}$ при $i \neq j \Rightarrow 3=\left(1+x_{i} y_{j}\right)\left(1+x_{j} y_{i}\right)=1+x_{i} y_{j}+x_{j} y_{i}+$ 4. Подставляя $y_{i}=\frac{2}{x_{i}}$, получаем $x_{i}^{2}+x_{i} x_{j}+x_{j}^{2}=0$. Значит, $x_{i}=\xi^{ \pm 1} x_{j}$, где $\xi=e^{(2 \pi i) / 3}$. Сопрягая матрицей $\operatorname{diag}\left(1, x_{1}\right)$, устанавливаем, что каждый сбалансированный базис сопряжен базису, в котором $x_{1}=1$. Отсюда $x_{2}=\xi$, $x_{3}=\xi^{2}$ или $x_{2}=\xi^{2}, x_{3}=\xi$. В обоих случаях мы имеем один и тот же базис. Теорема доказана.

Если потребовать, чтобы сбалансированный базис в $\mathrm{M}_{2}$ был инвариантен относительно группы $H$, то таких базисов будет два: $\mathscr{B}_{2}^{+}$и $\mathscr{B}_{2}^{-}$. Они являются дополнительными:

$$
\mathscr{B}_{2}^{ \pm}=\left\{\frac{1}{2}\left(E_{2} \pm A_{x}\right): x \in \mathbb{F}_{2}^{2}\right\}, \quad \text { где } \quad A_{x}=\frac{1}{\sqrt{3}} \sum_{u \neq 0}(-1)^{\langle x \mid u\rangle} i^{B(u, u)} J_{u}
$$

Здесь $i^{a}=i$, если $a=1$, и $i^{a}=1$, если $a=0$. Отметим, что, в отличие от случая $p>2$, группа Aut $\mathscr{B}_{2}^{ \pm}$не совпадает с $N(J)$, а является в ней подгруппой индекса 2.

Tеорема 3. В алгебре $\mathrm{M}_{2^{n}}(\mathbb{C})$ nри $n>1$ не существует сбалансированного базиса ранга $r$, обладающего регулярной әлементарной абелевой 2-группой автоморфизмов, если $\frac{r\left(2^{n}-r\right)}{2^{2 n}-1}$ не является квадратом рационального числа.

ДокАЗАТЕЛЬство. Пусть сбалансированный базис $\mathscr{B}$ в $\mathrm{M}_{2^{n}}, n>1$, обладает регулярной элементарной абелевой 2-группой автоморфизмов $H_{0}$. Тогда из транзитивности $H_{0}$ на $\mathscr{B}$ следует (проективная) неприводимость $H_{0}$. Как доказано в [6], неприводимые конечные абелевы подгруппы в Aut $\mathrm{M}_{n}$ сопряжены в Aut $\mathrm{M}_{n}$, если они изоморфны. Следовательно, $H_{0}$ сопряжена с $H$. Поэтому мы можем считать, что $H_{0}=H \leqslant$ Aut $\mathscr{B}$.

Положим $q=2^{n}>2$. Находим параметры 2-системы $\mathscr{B}: k=r q, \lambda=\frac{(r q-1) r}{q^{2}-1}$.

Для $a \in \mathbb{F}_{2}^{2 n}$, как и раньше, определим $i^{a}=i$, если $a=1$, и $i^{a}=1$, если $a=0$. При этом справедливы равенства

$$
i^{a+b}=i^{a} i^{b}(-1)^{a b} \text { и } i^{a} i^{a}(-1)^{a}=1 .
$$

Пусть $e_{0}=\sum_{u} \alpha_{u} i^{B(u, u)} J_{u} \in \mathscr{B}$, тогда

$$
e_{x}=J_{x} e_{0} J_{x}^{-1}=\sum_{u} \alpha_{u}(-1)^{\langle x \mid u\rangle} i^{B(u, u)} J_{u} \in \mathscr{B}
$$


Заметим, что $\left(i^{B(u, u)} J_{u}\right)^{2}=J_{0}=E_{q}$ - единичная матрица порядка $q$. Так как $\operatorname{tr} J_{u}=0$ при $u \neq 0$, то

$$
\operatorname{tr} e_{0}^{2}=q \sum_{u} \alpha_{u}^{2}=\operatorname{tr} e_{0}=r, \quad \operatorname{tr} e_{0} e_{x}=q \sum_{u} \alpha_{u}^{2}(-1)^{\langle x \mid u\rangle}=\lambda
$$

для всех $0 \neq x \in \mathbb{F}_{2}^{2 n}$. Таким образом, мы имеем линейную систему (4.1) относительно неизвестных $\alpha_{u}^{2}$ с квадратной матрицей коэффициентов $S=\left(s_{x, u}\right)_{q^{2} \times q^{2}}$, $s_{x, u}=(-1)^{\langle x \mid u\rangle}$. Матрица $S$, очевидно, является таблицей неприводимых характеров элементарной абелевой 2-групшы ранга $2 n$. Поэтому $S^{t} S=q^{2} E_{q^{2}}$. Следовательно,

$$
\begin{array}{r}
S \cdot\left(\alpha_{0}^{2}, \ldots, \alpha_{u}^{2}, \ldots\right)^{t}=\frac{1}{q}(r, \lambda, \ldots, \lambda)^{t} \Rightarrow\left(\alpha_{0}^{2}, \ldots, \alpha_{u}^{2}, \ldots\right)^{t} \\
\quad=\frac{1}{q^{3}} S^{t} \cdot(r, \lambda, \ldots, \lambda)^{t} \Rightarrow \alpha_{0}^{2}=\frac{1}{q^{3}}\left(r+\lambda\left(q^{2}-1\right)\right)=\frac{r^{2}}{q^{2}} \\
\alpha_{u}^{2}=\frac{1}{q^{3}}\left(r+\sum_{x \neq 0}(-1)^{\langle x \mid u\rangle} \lambda\right)=\frac{1}{q^{3}}(r-\lambda)=\frac{r(q-r)}{q^{2}\left(q^{2}-1\right)} .
\end{array}
$$

Так как $\operatorname{tr} e_{0}=\operatorname{tr} \alpha_{0} J_{0}=\alpha_{0} q$, то $\alpha_{0}=r / q$. Положим

$$
\alpha_{u}=(-1)^{f(u)} \frac{\alpha}{q}, \quad \text { где } \alpha=\sqrt{\frac{r(q-r)}{q^{2}-1}} .
$$

Далее, приравнивая в равенстве $e_{0}=e_{0}^{2}$ коэффициенты при $J_{v}, v \neq 0$, получаем

$$
\begin{aligned}
& \alpha_{v} i^{B(v, v)}=\sum_{u} \alpha_{u} \alpha_{u+v} i^{B(u, u)} i^{B(u+v, u+v)}(-1)^{B(u, u+v)}=2 \alpha_{0} \alpha_{v} i^{B(v, v)} \\
& \quad+\sum_{u \neq 0, v} \alpha_{u} \alpha_{u+v} i^{B(u, u)} i^{B(u+v, u+v)}(-1)^{B(u, u+v)}=\frac{2 r}{q^{2}} \alpha(-1)^{f(v)} i^{B(v, v)} \\
& \quad+\sum_{u \neq 0, v} \frac{\alpha^{2}}{q^{2}}(-1)^{f(u)+f(u+v)+B(u, u+v)} i^{B(u, u)} i^{B(u+v, u+v)} .
\end{aligned}
$$

Отсюда следует

$$
0=(2 r-q)(-1)^{f(v)} i^{B(v, v)}+\alpha \sum_{u \neq 0, v} \kappa(u, v),
$$

где $\kappa(u, v)=(-1)^{f(u)+f(u+v)+B(u, u+v)} i^{B(u, u)} i^{B(u+v, u+v)}$.

Если $\langle u \mid v\rangle=1$, то $\kappa(u, v)=-\kappa(u+v, v)$. Положим $V_{1}=\{u:\langle u \mid v\rangle=1\}$. Тогда $u \in V_{1} \Leftrightarrow u+v \in V_{1}$. Следовательно, разбивая $V_{1}$ на пары $\{u, u+v\}$, получаем $\sum_{u \in V_{1}} \kappa(u, v)=0$.

Пусть $V_{0}=\{u:\langle u \mid v\rangle=0, u \neq 0, v\}$. Тогда $\left|V_{0}\right|=2^{2 n-1}-2>0$, так как $n>1$. Далее, $u \in V_{0} \Leftrightarrow u+v \in V_{0}$ и

$$
\begin{aligned}
\kappa(u, v) & =\kappa(u+v, v)=(-1)^{f(u)+f(u+v)} i^{B(u, u)} i^{B(u+v, u+v)}(-1)^{B(u, u+v)} \\
& =(-1)^{f(u)+f(u+v)} i^{B(u, u)} i^{B(u, u)} i^{B(v, v)}(-1)^{B(u, u) B(v, v)}(-1)^{B(u, u)+B(u, v)} \\
& =(-1)^{f(u)+f(u+v)+B(u, u) B(v, v)+B(u, v)} i^{B(v, v)}=\tau(u, v) i^{B(v, v)} .
\end{aligned}
$$


Если $u \in V_{0}$, то $\tau(u, v)=\tau(u+v, v)= \pm 1$. Значит, $V_{0}$ разбивается на нечетное число $2^{2 n-2}-1$ пар $\{u, u+v\}$ и на каждой паре $\tau$ принимает значение +1 или -1 . Следовательно, $\sum_{u \in V_{0}} \tau(u, v) \neq 0$ и в силу $(4.2) \alpha=\sqrt{\frac{r(q-r)}{q^{2}-1}} \in \mathbb{Q}$. Теорема доказана.

СлеДСТВИЕ 1. В алгебре $\mathrm{M}_{2^{n}}(\mathbb{C}), n>1$, не существует сбалансированных базисов ранга $r$, обладающих регулярной әлементарной абелевой 2-группой автоморфизмов, если выполняется одно из следующих условий:

1) $r=1$ или $r=2^{n}-1, n \neq 3$;

2) $r=2^{m}$, әде $m \geqslant 1$.

Перед рассмотрением теоретико-числовых вопросов введем стандартные обозначения: $a \mid b$ означает $a$ делит $b ;(a, b)$ обозначает наибольший общий делитель $a$ и $b$.

Для доказательства следствия нам понадобится лемма, первые три пункта которой хорошо известны, а четвертый, возможно, представляет новое утверждение, имеюшее самостоятельный интерес.

\section{ЛЕмма 1.}

(i) Уравнение $2^{x}+1=y^{2}$ имеет единственное решение в натуральных числах $x=y=3$;

(ii) уравнение $2^{x}-1=y^{2}$ имеет единственное решение в натуральных числах $x=y=1$;

(iii) $\left(2^{n}-1,2^{m}-1\right)=2^{(n, m)}-1$;

(iv) число $1+2^{2 d}+\cdots+2^{2 n d}$ не является квадратом натурального числа ни при каких натуральных $d$ u $n$.

ДокаЗательство. (i) Имеем: $2^{x}=(y-1)(y+1) \Rightarrow(y-1) \mid(y+1) \Rightarrow(y-1)=$ $(y-1, y+1)$. Но $(y-1, y+1) \leqslant 2$. Значит, $y-1=2$ и $x=3$.

Пункт (ii) вытекает из того, что квадрат нечетного числа сравним с 1 по модулю 4 .

(iii) Пусть $a=\left(2^{n}-1,2^{m}-1\right)$ и $n>m$. Тогда $a \mid\left(2^{n}-1\right)-\left(2^{m}-1\right)=$ $2^{m}\left(2^{n-m}-1\right) \Rightarrow a \mid\left(2^{n-m}-1\right)$. Отсюда выводим $a \mid\left(2^{r}-1\right)$, где $r$ - остаток от деления $n$ на $m$. Теперь, используя алгоритм Евклида, получаем $a \mid\left(2^{(n, m)}-1\right)$. Значит, $a=2^{(n, m)}-1$.

(iv) Положим $A(n, d)=1+2^{2 d}+\cdots+2^{2 n d}$. Рассмотрим отдельно два случая - когда $n$ четно и когда $n$ нечетно.

Случай 1. $n=2 m-1$. Имеем

$A(n, d)=\frac{2^{2(n+1) d}-1}{2^{2 d}-1}=\frac{2^{4 m d}-1}{2^{2 d}-1}=\frac{2^{2 m d}-1}{2^{2 d}-1} \cdot\left(2^{2 m d}+1\right)=A(m-1, d)\left(2^{2 m d}+1\right)$.

Очевидно, $\left(2^{2 m d}-1,2^{2 m d}+1\right)=1 \Rightarrow\left(A(m-1, d), 2^{2 m d}+1\right)=1$. Значит, так как в силу п. (i) $2^{2 m d}+1-$ не квадрат, то $A(n, d)-$ не квадрат.

Случай 2. $n=2 m$. Имеем

$$
\begin{aligned}
A(n, d) & =\frac{2^{2(2 m+1) d}-1}{2^{2 d}-1}=\frac{2^{(2 m+1) d}-1}{2^{d}-1} \cdot \frac{2^{(2 m+1) d}+1}{2^{d}+1} \\
& =\left(1-2^{d}+2^{2 d}-\cdots+2^{2 m d}\right)\left(1+2^{d}+2^{2 d}+\cdots+2^{2 m d}\right) .
\end{aligned}
$$


Положим $a=1-2^{d}+2^{2 d}-\cdots+2^{2 m d}, b=1+2^{d}+2^{2 d}+\cdots+2^{2 m d}$. Так как $\left(2^{(2 m+1) d}-1,2^{(2 m+1) d}+1\right)=1$, то $(a, b)=1$. Будем рассуждать от противного. Предположим, что $A(n, d)$ - квадрат. Тогда $a=a_{1}^{2}, b=b_{1}^{2}$. Если $d=1$, то $a \equiv b \equiv-1(\bmod 4)$. Это противоречит тому, что $a$ и $b-$ квадраты. Поэтому далее считаем $d>1$.

Имеем $b_{1}^{2}-a_{1}^{2}=2^{d+1}\left(1+2^{2 d}+\cdots+2^{2(m-1) d}\right)$. Так как $b_{1}, a_{1}$ - нечетные взаимопростые числа, то $\left(b_{1}-a_{1}, b_{1}+a_{1}\right)=2$. Значит,

$$
\text { либо (1) }\left\{\begin{array} { l } 
{ b _ { 1 } - a _ { 1 } = 2 x , } \\
{ b _ { 1 } + a _ { 1 } = 2 ^ { d } y , }
\end{array} \text { либо } ( 2 ) \quad \left\{\begin{array}{l}
b_{1}-a_{1}=2^{d} x, \\
b_{1}+a_{1}=2 y,
\end{array}\right.\right.
$$

где $x, y$ - нечетные взаимно простые числа.

Системы (1) и (2) переводятся друг в друга заменами $a_{1} \leftrightarrow-a_{1}, x \leftrightarrow y$. Так как у нас был произвол в выборе знака $a_{1}$, то достаточно рассмотреть систему (1).

Имеем

$$
x y=1+2^{2 d}+\cdots+2^{2(m-1) d}=\frac{2^{2 m d}-1}{2^{2 d}-1} .
$$

Кроме того, равенства $b_{1}=x+2^{d-1} y, a_{1}=-x+2^{d-1} y$ влекут

$$
x^{2}+2^{2 d-2} y^{2}=\frac{1}{2}\left(b_{1}^{2}+a_{1}^{2}\right)=1+2^{2 d}+\cdots+2^{2 m d} .
$$

Из (4.3) и (4.4) получаем $x^{2}+2^{2 d-2} y^{2}=x y+2^{2 m d}$. В силу (4.3) $2^{2 m d}=1+$ $\left(2^{2 d}-1\right) x y$. Отсюда следует $x^{2}+2^{2 d-2} y^{2}=x y+1+\left(2^{2 d}-1\right) x y \Rightarrow x^{2}-2^{2 d} x y+$ $2^{2 d-2} y^{2}=1 \Rightarrow$

$$
\left(x-2^{2 d-1} y\right)^{2}-\left(2^{4 d-2}-2^{2 d-2}\right) y^{2}=1 .
$$

Так как $2^{4 d-2}-2^{2 d-2}=2^{2 d-2}\left(2^{2 d}-1\right)$ не является квадратом в силу п. (ii), то поле $F=\mathbb{Q}\left[\sqrt{2^{2 d}-1}\right]$ суть квадратичное расширение поля рациональных чисел $\mathbb{Q}$. Равенство (4.5) означает, что норма числа $z=x-2^{2 d-1} y+y \sqrt{2^{4 d-2}-2^{2 d-2}}$, принадлежащего порядку $\mathscr{P}=\left\langle 1, \sqrt{2^{4 d-2}-2^{2 d-2}}\right\rangle_{\mathbb{Z}}$ поля $F$, равна 1 . Значит, $z-$ единица (= обратимый элемент) порядка $\mathscr{P}$. В силу известной теоремы Дирихле о строении группы единиц порядков полей алгебраических чисел, примененной к вешественному квадратичному расширению поля $\mathbb{Q}, z= \pm \varepsilon^{k}$, где $\varepsilon$ - основная единица порядка $\mathscr{P}_{\text {и }} k \in \mathbb{Z}$. Условие $\varepsilon>1$ определяет основную единицу однозначно. Найдем $\varepsilon$ методом разложения числа $\sqrt{2^{4 d-2}-2^{2 d-2}}$ в цепную дробь (см. [8]):

$$
\begin{aligned}
\sqrt{2^{4 d-2}-2^{2 d-2}} & =2^{2 d-1}-1+\left(\sqrt{2^{4 d-2}-2^{2 d-2}}-2^{2 d-1}+1\right), \\
\frac{1}{\sqrt{2^{4 d-2}-2^{2 d-2}}-2^{2 d-1}+1} & =1+\frac{\sqrt{2^{4 d-2}-2^{2 d-2}}-2^{2 d-2}}{2^{2 d}-2^{2 d-2}-1} \\
\frac{2^{2 d}-2^{2 d-1}-1}{\sqrt{2^{4 d-2}-2^{2 d-2}}-2^{2 d-2}} & =2+\frac{\sqrt{2^{4 d-2}-2^{2 d-2}}-2^{2 d-2}}{2^{2 d-2}} \\
\frac{2^{2 d-2}}{\sqrt{2^{4 d-2}-2^{2 d-2}}-2^{2 d-2}} & =1+\frac{\sqrt{2^{4 d-2}-2^{2 d-2}}-2^{2 d-1}+1}{2^{2 d-1}+2^{2 d-2}-1} .
\end{aligned}
$$

Одновременно заполняем таблицу, в которой $q_{s}$ - неполные частные, $\frac{P_{s}}{Q_{s}}-$ подходящие дроби. При этом $P_{s}=q_{s} P_{s-1}+P_{s-2}, Q_{s}=q_{s} Q_{s-1}+Q_{s-2}$ и $P_{-1}=1$, 
$Q_{-1}=0, P_{0}=q_{0}, Q_{0}=1$. Первая встретившаяся в последней строке таблицы \pm 1 будет означать, что числитель и знаменатель соответсвующей подходящей дроби дают искомую основную единицу $\varepsilon=P_{s}+Q_{s} \sqrt{2^{4 d-2}-2^{2 d-2}}$. При этом в нашем случае должна появиться именно 1 , так как очевидно, что норма любой единицы в $\mathscr{P}$ сравнима с 1 по модулю 4.

\begin{tabular}{|c|c|c|c|c|}
\hline$s$ & 0 & 1 & 2 & 3 \\
\hline$q_{s}$ & $2^{2 d-1}-1$ & 1 & 2 & 1 \\
\hline$P_{s}$ & $2^{2 d-1}-1$ & $2^{2 d-1}$ & $2^{2 d}+2^{2 d-1}-1$ & $2^{2 d+1}-1$ \\
\hline$Q_{s}$ & 1 & 1 & 3 & 4 \\
\hline$P_{s}^{2}-\left(2^{4 d-2}-2^{2 d-2}\right) Q_{s}^{2}$ & $-2^{2 d}+2^{2 d-2}+1$ & $2^{2 d-2}$ & $-2^{2 d}+2^{2 d-2}+1$ & 1 \\
\hline
\end{tabular}

Так как $d>1$, то 1 впервые появляется в последней строке таблицы при $s=3$. Значит, $\varepsilon=2^{2 d+1}-1+4 \sqrt{2^{4 d-2}-2^{2 d-2}}-$ основная единица порядка $\mathscr{P}$. Легко видеть, что тогда для произвольной единицы $u+v \sqrt{2^{4 d-2}-2^{2 d-2}} \in \mathscr{P}$ коэффициент $v$ делится на 4 . Следовательно, $z=x-2^{2 d-1} y+y \sqrt{2^{4 d-2}-2^{2 d-2}}$ не может быть единицей, так как $y$ нечетно. Противоречие доказывает (iv). Лемма доказана.

ДоКАЗАТЕЛЬСТво СЛЕДСТВИЯ 1 . Положим $Q(r, n)=\frac{r\left(2^{n}-r\right)}{2^{2 n}-1}$. В силу теоремы 3 достаточно доказать, что $Q(r, n)$ не является квадратом при ограничениях, наложенных на $r$ и $n$.

1) Имеем: $Q(1, n)=Q\left(2^{n}-1, n\right)=\frac{1}{2^{n}+1}-$ не квадрат при $n \neq 3$ в силу п. (i) леммы 1.

2) Имеем: $Q\left(2^{m}, n\right)=\frac{2^{2 m}\left(2^{n-m}-1\right)}{2^{2 n}-1}$. Положим $n-m=l$ и $d=(l, 2 n)$. Отметим, что $l<n$. Очевидно, $Q\left(2^{m}, n\right)-$ не квадрат $\Leftrightarrow \frac{2^{l}-1}{2^{2 n}-1}-$ не квадрат. Рассмотрим два случая.

Случай 1. $l$ нечетно. Имеем $d \mid n$. Положим $n=d n_{1}, l=d l_{1}$. Тогда после сокращения на $2^{d}-1$ получаем

$$
\frac{2^{l}-1}{2^{2 n}-1}=\frac{1+2^{d}+\cdots+2^{d\left(l_{1}-1\right)}}{\left(1+2^{d}+\cdots+2^{d\left(n_{1}-1\right)}\right)\left(2^{n}+1\right)},
$$

где последняя дробь несократима в силу п. (iii) леммы 1 и в ее знаменателе стоят взаимно простые множители, так как $\left(2^{n}-1,2^{n}+1\right)=1$. Следовательно, по п. (i) леммы 1 дробь $\frac{2^{l}-1}{2^{2 n}-1}$ может быть квадратом только при $n=3$. Но тогда $2^{3}-1=7 \mid\left(2^{l}-1\right)$, что невозможно, ибо $l<n=3$.

Случай 2. $l$ четно. Тогда $d$ четно. Положим $d=2 d_{1}, n=d_{1} n_{1}, l=d l_{1}$. После сокращения на $2^{d}-1$ получим

$$
\frac{2^{l}-1}{2^{2 n}-1}=\frac{1+2^{2 d_{1}}+\cdots+2^{2\left(l_{1}-1\right) d_{1}}}{1+2^{2 d_{1}}+\cdots+2^{2\left(n_{1}-1\right) d_{1}}},
$$

где последняя дробь несократима по п. (iii) леммы 1 и, следовательно, не может быть квадратом в силу п. (iv) леммы 1. Следствие доказано. 
ЗАмечАния. 1) Доказательство несуществования сбалансированного $H$-инвариантного базиса ранга $2^{n-1}$ в $\mathrm{M}_{2^{n}}, n>1$, не требует привлечения теоретико-числовых рассмотрений и следует из формулы (4.2), так как в этом случае $2 r-q=0$.

2) По-видимому, дробь $\frac{r\left(2^{n}-r\right)}{2^{2 n}-1}$ является квадратом рационального числа только при $n=3$ и $r=1$ или 7 . Однако автор пока не располагает доказательством этого утверждения. Также неизвестно, существует ли сбалансированный базис ранга 1 в $\mathrm{M}_{8}(\mathbb{C})$, допускающий регулярную элементарную абелеву 2-группу автоморфизмов.

\section{§5. Сбалансированные $1-(n+1, r, r n)$-системы}

В [2] доказано, что существует единственная с точностью до сопряженности 1 - $(n+1,1, n)$-система $\mathscr{E}(n)$. При этом $\mathscr{E}(n)$ является фактически $(n+1)-(n+1,1, n)$ системой и Aut $\mathscr{E}(n) \cong S_{n+1}-$ симметрическая группа. Система $\mathscr{E}(n)$ состоит из идемпотентов

$$
\begin{gathered}
f_{i}=(0, \ldots, 1, \ldots, 0)^{t} \cdot\left(-\frac{1}{n}, \ldots, 1, \ldots,-\frac{1}{n}\right) \quad(1 \text { на } i \text {-й позиции }), \quad i=1, \ldots, n, \\
f_{n+1}=(1, \ldots, 1)^{t} \cdot\left(\frac{1}{n}, \ldots, \frac{1}{n}\right) .
\end{gathered}
$$

Положим $\mathscr{E} \otimes E_{r}=\left\{e \otimes E_{r}: e \in \mathscr{E}\right\}$.

ТЕОРема 4. В алгебре $\mathrm{M}_{r n}$ каждая 1- $(n+1, r, r n)$-система ह сопряжена системе $\mathscr{E}(n) \otimes E_{r}$. В частности, $\mathscr{E}$ является $(n+1)-(n+1, r, r n)$-системой, порожсдающей подалгебру, изоморфную $\mathrm{M}_{n}, u$ Aut $\mathscr{E} \cong S_{n+1} \times G L(r, \mathbb{C})$.

ДокАЗАТЕЛьство. Пусть $\mathscr{E}=\left\{e_{1}, \ldots, e_{n+1}\right\}$ - сбалансированная $1-(n+1$, $r, r n)$-система в алгебре $\mathrm{M}_{r n}$. Из условия сбалансированности, применяя к обеим частям функцию $\mathrm{Tr}$, получаем $(n+1) r=k r n$. Отсюда находим параметр $k=$ $(n+1) / n$.

Как показано в [2], каждый идемпотент ранга 1 может быть представлен в виде $e_{i}=X^{(i)} Y_{i}$, где $X^{(i)}, Y_{i}$ - матрицы ранга $r$ размеров соответственно $r n \times r$ и $r \times r n$. Такое представление не единственно. Любое другое представление может быть получено из данного единственным образом с помощью подходящей невырожденной $(r \times r)$-матрицы $T: e_{i}=X^{(i)} T \cdot T^{-1} Y_{i}$. Отметим, что

$(*)$ если $j$-я строка матрицы $X^{(i)}$ ненулевая, то, используя метод исключения Гаусса, можно подобрать матрицу $T$ так, чтобы $j$-я строка матрицы $X^{(i)} T$ состояла из нулей и одной единицы в любой наперед заданной позиции.

Шаг 1. Положим $X=\left(X^{(1)} \ldots X^{(n+1)}\right)$. Покажем, что ранг матрицы $X(i)=$ $\left(X^{(1)} \ldots \widehat{X^{(i)}} \ldots X^{(n+1)}\right)$, полученной из $X$ вычеркиванием $X^{(i)}$, равен $r n$.

Достаточно рассмотреть случай $i=n+1$. Предположим, что $\operatorname{rank} X(n+1)$ $<r n$. Тогда сопряжением в $\mathrm{M}_{r n}$ можно добиться, чтобы последняя строка матриц $X^{(i)}, i=1, \ldots, n$, была нулевой. Далее, последнюю строку матрицы $X^{(n+1)}$, которая в силу условия сбалансированности нулевой быть не может, без ограничения общности в силу $(*)$ можно считать равной $(0, \ldots, 0,1)$. Тогда из условия 
сбалансированности

$$
\sum_{i=1}^{n}\left(\begin{array}{ccc}
* & \ldots & * \\
\ldots & \ldots & \ldots \\
* & \ldots & * \\
0 & \ldots & 0
\end{array}\right) Y_{i}+\left(\begin{array}{cccc}
* & \ldots & * & * \\
\ldots & \ldots & \ldots & \ldots \\
* & \ldots & * & * \\
0 & \ldots & 0 & 1
\end{array}\right) Y_{n+1}=k E_{r n}
$$

следует, что последняя строка матрицы $Y_{n+1}$ равна $(0, \ldots, 0, k)$. Так как условие идемпотентности $e_{n+1}^{2}=e_{n+1}$ влечет $E_{r}=Y_{n+1} X^{(n+1)}$, то, приравнивая последние строки матриц $E_{r}$ и $Y_{n+1} X^{(n+1)}$, получаем $k=1$. Это противоречит найденному ранее значению $k=(n+1) / n$.

Шаг 2. Так как $\operatorname{rank} X(n+1)=r n$, то, переходя к сопряженной системе $X^{-1}(n+1) \mathscr{E} X(n+1)$, получаем, что без ограничения общности можно считать $X(n+1)=E_{r n}$. Отсюда в силу равенства $Y_{i} X^{(i)}=E_{r}$ выводим

$$
X^{(i)}=\left(\begin{array}{c}
0 \\
\vdots \\
E_{r} \\
\vdots \\
0
\end{array}\right), \quad Y_{i}=\left(A_{i 1} \ldots A_{i i-1} E_{r} A_{i i+1} \ldots A_{i n}\right), \quad i=1, \ldots, n, \quad A_{i j} \in \mathrm{M}_{r}
$$

Положим

$$
X^{(n+1)}=\left(\begin{array}{c}
B_{1} \\
\vdots \\
B_{n}
\end{array}\right), \quad Y_{n+1}=\left(C_{1} \ldots C_{n}\right), \quad B_{i}, C_{i} \in \mathrm{M}_{r}
$$

Тогда условие сбалансированности

$$
\sum_{i=1}^{n} X^{(i)} Y_{i}+X^{(n+1)} Y_{n+1}=\frac{n+1}{n} E_{r n}
$$

записывается в виде

$$
\left(\begin{array}{cccc}
E_{r} & A_{12} & \ldots & A_{1 n} \\
A_{21} & E_{r} & \ldots & A_{2 n} \\
\ldots \ldots & \ldots & \ldots & \ldots \\
A_{n 1} & A_{n 2} & \ldots & E_{r}
\end{array}\right)+\left(\begin{array}{c}
B_{1} \\
\vdots \\
B_{n}
\end{array}\right) \cdot\left(C_{1} \ldots C_{n}\right)=\frac{n+1}{n} E_{r n}
$$

Приравнивая в (5.1) диагональные блоки, получаем: $B_{i} C_{i}=\frac{1}{n} E_{r}$. Для недиагональных блоков имеем: $\left(A_{i j}+B_{i} C_{j}=0\right) \Rightarrow\left(A_{i j}=\frac{1}{n} B_{i} B_{j}^{-1}\right)$. Сопрягая блочно-диагональной матрицей $T=\operatorname{diag}\left(B_{1}^{-1}, \ldots, B_{n}^{-1}\right)$, получаем $T e_{i} T^{-1}=f_{i} \otimes E_{r}$, т.е. $\mathscr{E}$ сопряжена системе $\mathscr{E}(n) \otimes E_{r}$. Теорема доказана. 


\section{Зак лючение}

В заключение отметим два дальнейших направления исследований. Как известно, в комбинаторном анализе широкое изучение получили симметричные блоксхемы, обладаюшие регулярной группой автоморфизмов. Это то, что назьвается разностными множествами в группах. Интересен некоммутативный аналог этой тематики.

ПроБЛемА 1. Классифицировать сбалансированнье базисы в некоммутативных алгебрах (в частности, в алгебрах матрич), допускающие регулярную (абелеву) группу автоморфизмов.

В работе [2] приводится бесконечное семейство попарно несопряженных $2-(6,1,3)$-систем. Тем не менее автору кажется правдоподобной

ГипотезА 1. В любой алгебре существует не более чем конечное число попарно несопряженных сбалансированных базисов.

\section{Список литературы}

1. Иванов Д. Н. Ортогональные разложения ассоциативных алгебр и сбалансированные системы идемпотентов // Матем. сб. 1998. Т. 189. № 12. С. 83-102.

2. Иванов Д. Н. Сбалансированные системы из примитивных идемпотентов в алгебрах матриц // Матем. сб. 2000. Т. 191. № 4. С. 67-90.

3. Холл М. Комбинаторика. М.: Мир, 1970.

4. Кострикин А.И., Кострикин И. А., Уфнаровский В. А. Ортогоналњные разложения простых алгебр Ли (тип $\left.A_{n}\right)$ // Труды МИАН. 1981. Т. 158. С. $105-120$.

5. Conway J.H., Curtis R. T., Norton S.P., Parker R.A., Wilson R.A. Atlas of finite groups. Oxford: Clarendon Press, 1985.

6. Супруненко Д. А. Группы матриц. М.: Наука, 1979.

7. Kostrikin A.I., Pham Huu Tiep. Orthogonal decompositions and integral lattices. Berlin: Walter de Gruyter, 1994.

8. Боревич З. И., Шафаревич И. Р. Теория чисел. М.: Наука, 1972.

Московский государственньй

Поступила в редакцию

университет им. М. В. Ломоносова

15.03 .2000

E-mail: Dmitry.Ivanov@tversu.ru 